\title{
Desafíos en la formación de los periodistas españoles: convergencia europea, capacitación tecnológica y formación permanente
}

\author{
Challenges in the formation of the Spanish journalists: European \\ convergence, technological training and permanent professional \\ formation
}

\author{
Pilar Sánchez \\ Universidad de Valladolid \\ pilar.sanchez@hmca.uva.es
}

\begin{abstract}
Resumen
La formación de los periodistas en España se encuentra en pleno proceso de cambio por su reciente adaptación a los programas de convergencia europea y por la revolución que sufre el sector con la llegada de las nuevas tecnologías de la información y de la comunicación dentro y fuera de las aulas. Este artículo parte del debate aún abierto en el ámbito académico y profesional en torno a la pertinencia de la formación universitaria de los periodistas con una aproximación a la historia de la enseñanza del periodismo en España y su tardía incorporación a su formación universitaria. Se trata de una revisión historicista que pretende ayudar a entender el estado actual de la titulación tras la llegada de las nuevas tecnologías que plantean desafíos concretos: la necesidad de una preparación multimedia integral de los periodistas que vaya más allá de los soportes técnicos y la exigencia de una formación permanente.
\end{abstract}

Palabras Clave: Universidad, periodismo, ciberperiodismo, formación, EEES.

\begin{abstract}
The professional formation of the journalists in Spain is currently changing due to its recent adaptation to European convergence programs and also because of the revolution that the sector is facing because of the advent of new information and communication technologies inside and outside the classroom. This article is part of the still opened debate in the academic and professional area concerning the relevancy of university education of the journalists with an approach to history of the education of the Journalism in Spain and its late incorporation into
\end{abstract}


the university. It is a question of a historicist review that tries helping to deal the current state of the qualifications after the arrival of the new technologies that raise specific challenges: the need of a multimedia preparation of the journalists that goes beyond the technical supports and the exigency of a permanent training.

Keywords: University, journalism, cyberjournalism, formation, EEES.

\section{CONTEXTO ACTUAL Y MARCO TEÓRICO}

El ejercicio del periodismo se encuentra en una encrucijada económica y tecnológica que ha removido los cimientos del sector en un ámbito mundial. A la búsqueda de nuevos modelos de negocio, se unen nuevos perfiles laborales y exigencias del mercado que afectan directamente a la formación que reciben los periodistas. La revisión del estado de la profesión pasa necesariamente por una ineludible reflexión sobre la preparación universitaria de los informadores encaminada a proporcionar mayor flexibilidad ante un mercado profesional polivalente, cambiante y multimedia.

La situación en España no es diferente a la de los países de su entorno europeo que han implantado recientemente nuevos planes de estudio (curso 2010-2011) adaptados al llamado Espacio Europeo de Educación Superior (EEES) con el que se trata de unificar los títulos universitarios y los criterios de evaluación para facilitar la movilidad laboral entre países. Un cambio de programaciones que coincide con la incorporación de nuevas tecnologías en las aulas para la capacitación de los periodistas en nuevos soportes multimedia.

En esta coyuntura concurren motivos de interés y oportunidad para plantear el objetivo de esta investigación, centrada en la revisión del estado actual de la enseñanza del periodismo en España, a través de los planes de estudio vigentes, que afronta desafíos concretos: la convergencia europea de los estudios superiores, la preparación multimedia integral y la formación permanente de los periodistas.

En el caso español debe tenerse en cuenta la perspectiva de la tardía incorporación de los periodistas a la Universidad, que cuenta con poco más de 40 años de historia, en la que influyó la censura y el control informativo de la dictadura Franquista (19391975). En este contexto, sigue abierto el debate sobre la pertinencia de esta enseñanza superior, avivado por la inexistencia de una normativa que exija el título universitario para ejercer la profesión.

Las principales investigaciones sobre los estudios del periodismo en España comenzaron en el siglo XX, destacando los trabajos de Manuel Graña (1927), Juan Beneyto (1958) o Ángel Benito (1967), defensores de la enseñanza universitaria e integral del periodista. Una vez que en 1971 los estudios se incorporaron a la Universidad española destacaron los análisis de Enrique de Aguinaga (1984), 
Marisa Aguirre (1988), Mercedes Gordon, (1991), María Luisa Humanes (1997) y posteriormente José Juan Videla (2002) y Elena Real (2004).

A estos trabajos se añaden los estudios recientes sobre el cambio formativo con la incorporación de las nuevas tecnologías en las aulas, destacando las investigaciones de Ramón Salaverría (2000), Javier Díaz Noci (2002), Koldo Meso (2003) o Santiago Tejedor (2007), entre otros. Sobre la adaptación al Espacio Europeo de Educación Superior, los análisis aún son escasos e incipientes, quizá por la falta de perspectiva tras la reciente incorporación de los nuevos planes educativos en el curso 2010-2011. Pero en España ya se han organizado congresos que han dado lugar a publicaciones sobre el tema, como es el caso de las dos más actuales coordinadas por Javier Sierra (2010), con la participación de diferentes autores.

Las investigaciones en las que se apoya este artículo, mediante una metodología cualitativa de corte historicista y bibliográfica, permiten construir el relato histórico de la formación de los periodistas en España, el devenir de unas enseñanzas que ayuda a entender los desafíos actuales de una profesión que requiere una formación integral y en permanente actualización.

\section{DEFENSORES Y DETRACTORES DE LA FORMACIÓN DE LOS PERIODISTAS EN LA UNIVERSIDAD}

La pertinencia de la formación universitaria de los periodistas sigue en cuestión en determinados sectores. Esta circunstancia ha condicionadola historia de su enseñanza en España y su consiguiente desarrollo científico puesto que no se incorporó a la Universidad hasta 1971. En ello influyó el control informativo y la censura de la Dictadura Franquista (1939-1975) y la concepción del periodismo como un oficio que requiere de cualidades innatas y práctica. Frente a esta postura está la de quienes defienden la visión de una profesión con necesidades de alta cualificación. Posición que, en gran medida, se ha impuesto.

Desde principios del siglo XX hubo investigadores y académicos que se atrevieron a defender la formación universitaria del periodista, entre los que destacó Manuel Graña, uno de los primeros profesores de Periodismo españoles perteneciente a la Escuela "El Debate":

No hay más remedio que admitir que existe la profesión de periodista, y que esa profesión, como todas, exige una vocación y aptitudes particulares, que se ensayan y perfeccionan en un aprendizaje más o menos lentos y más o menos metódico $[\ldots]$. Lo que desde luego parece chocante y difícil de explicar es que no se haya pensado antes en la formación técnica del periodista, en un aprendizaje escolar. Realmente es incompresible que haya, por ejemplo, una escuela de Veterinaria y no para una profesión cuyo recto juicio interesa a la sociedad (1926). 
El profesor Enrique de Aguinaga reivindica el paso de la concepción de la "actividad periodística” a la de "profesión periodística” porque, en su opinión, lo más relevante de este tránsito profesional dirigido hacia la Universidad es "la apertura de la investigación científica con carácter sistemático" (1980, p. 83).

Por otro lado, el Derecho a la Información es uno de los principales argumentos que esgrimen los defensores de la formación integral ya que los ciudadanos lo depositan en manos de los periodistas que adquieren un compromiso social y ético que requiere una sólida formación. Es un argumento fundamental en la tesis de la doctora Marisa Aguirre, entre otros, que presenta el acto informativo como "un acto de justicia” porque se da al ciudadano aquello a lo que tiene derecho. Y extiende este argumento a la idea de que "informar mal, por falta de formación o por el mal desempeño profesional, supone una injusticia” (1988, p. 40).

Igualmente, los defensores de esta enseñanza esgrimen que ante las presiones del mercado y los cambios tecnológicos, la formación universitaria proporciona al periodista mayor independencia (Aguirre, 1988) y sin olvidar que "el nivel de desarrollo en la capacitación de los profesionales informativos, viene a ser un índice del proceso de liberalización y de madurez de una sociedad" (Benito, 1982, p. 228).

Pero la defensa de estos estudios con rango universitario ha encontrado, igualmente, sus detractores. Las primeras iniciativas de formación de periodistas a través de escuelas privadas en las primeras décadas del siglo XX tropezaron con la oposición casi unánime de los propios periódicos españoles, que veían en ello un propósito de control por parte del Gobierno. Así se expresaba en un editorial del periódico de tirada nacional $\mathrm{ABC}$ :

Crear periodistas desde las aulas se nos antoja tan peregrino como hacer poetas desde una clase de Retórica o novelistas desde una empresa editorial [...] Si por añadidura se pretende instituir con una Escuela el título de periodista, entonces el proyecto puede llegar no sólo a lo absurdo, sino a lo grotesco (citado en Saiz y Seoane, 1996, p. 50).

Las primeras preocupaciones intelectuales sobre el periodismo giran en torno a los temas que trata y la influencia que ejerce. Es decir, no interesa tanto la preparación de los periodistas. Es a partir de los años 60 cuando la polémica se centra en la necesidad o no de una formación humanística o simplemente técnica. Así, comienza el debate sobre si el saber más técnico que se aprendía en el ejercicio rutinario de la profesión podía adquirirse mejor en algún centro donde la práctica del oficio estuviese acompañada de ciertos estudios técnicos y humanísticos.

El paso a la Universidad en los años 70 fue rechazado por los que temían que el cambio académico aportara una excesiva teorización y el alejamiento de la técnica. Un argumento utilizado por quienes consideran que la Universidad está alejada de la realidad proporcionando conocimientos puramente especulativos que no 
contribuyen a la adecuada preparación profesional (Iglesias, 1984).

Los detractores de la enseñanza superior utilizan, precisamente, el Derecho a la Información como el argumento principal de la libertad que debe existir en este sector que no admite regulación, titulación, ni etiquetas formativas. Recurren a dos falacias, en palabras de Elena Real:

Que la profesionalización del periodismo por medio de la titulación universitaria es una restricción de la libertad de expresión y particularmente del derecho reconocido y protegido en el artículo 20 de la Constitución española. Y que la profesionalización del periodismo por medio de la titulación universitaria es una forma de control inquisitorial de los periodistas por parte de los poderes gubernamentales (2004, p. 110).

En España, como en la mayoría de los países del entorno europeo, no se exige titulación universitaria para ser periodista y esto aún favorece el intrusismo y la defensa del oficio basada en la experiencia frente a la profesión con alta cualificación, pero la creciente demanda de estudiantes en las aulas evidencia que la formación del periodismo en la Universidad se impone.

\section{CUARENTA AÑOS DE PERIODISMO UNIVERSITARIO EN ESPAÑA}

La preocupación del director de un periódico español de principios de siglo XX por la formación de sus trabajadores supuso toda una revolución académica de un oficio que ahora se considera una Ciencia. Ángel Herrera Oria, jurista, periodista y sacerdote, fijó sin saberlo el punto de inflexión de la enseñanza de esta profesión en su Escuela de 'El Debate' que bautizó de forma homónima a la cabecera madrileña que él dirigió durante dos décadas. En la calle de la Colegiata de Madrid abrió en 1926 la sede de lo que se ha considerado la primera Escuela de Periodismo de España,"El Debate":

Defendía en solitario en la prensa de Madrid la necesidad de exigir para la práctica de la profesión determinados conocimientos y limpieza de conductas, fundó en 1926 su propia escuela, que funcionó hasta la Guerra Civil. Recibía en los años de la República muchas más solicitudes de las que podía atender, con un cupo establecido de 25 alumnos por curso" (Vigil y Vázquez, 1987, p. 7).

Como único precedente de este inicio formativo, cabe señalar el curso sobre periodismo que el catedrático y periodista Fernando Araujo Gómez organizó en Salamanca en 1887 y que algunos estudiosos consideran como un anticipo en toda Europa. Desde aquel momento, pasarían todavía casi tres décadas hasta que en España se pusiera en marcha la citada primera escuela para periodistas de "El Debate". Todo un 
hito académico el de 1926 que, sin embargo, contrasta con el dato de las 86 Facultades de Periodismo que ya existían en ese momento en EEUU.

Con el objetivo de dignificar la profesión periodística, Oria se inspiró en el ejemplo formativo pionero, el norteamericano de The School of Journalism de la Universidad de Columbia en Nueva York, fundada en 1912 por el magnate de la prensa norteamericana Joseph Pulitzer. Antes de abrir su escuela, Herrera Oria envió en 1920 a tres de sus discípulos a recibir formación: Manuel Graña, Francisco de Luis y Marcelino Oreja. Así, encendió los motores del nuevo centro de enseñanza periodística que supuso el embrión de los estudios universitarios en España.

De la escuela salieron siete directores de periódicos en apenas diez años, hasta que fue cerrada en 1936, justo al mismo tiempo que se producía el cierre del periódico, por orden del Gobierno de la República. Oria fundaría en 1960 la Escuela de Periodismo de la Iglesia. Y en ese paréntesis de la iniciativa privada, el Gobierno del dictador Francisco Franco creó la Escuela Oficial de Periodismo en noviembre de 1941 que dependía de la Delegación Nacional de Prensa y Propaganda, órgano considerado como el aparato censor del régimen, dirigido por Juan Aparicio, hasta que en 1951 pasó a depender del recién estrenado Ministerio de Información y Turismo. Su trayectoria contó con un marcado perfil político hasta que el ministro Manuel Fraga puso al frente al investigador y académico Juan Beneyto quien contribuyó a que estos estudios fueran adquiriendo un carácter universitario.

El Gobierno reconoció, a lo largo de los años, las distintas Escuelas de Periodismo que empezaron a florecer por toda la geografía nacional. Aquí se destacan las consideradas pioneras: el Instituto de Periodismo que en 1958 crea la Universidad de Navarra como el primer centro vinculado a la Universidad; en 1960, la citada Escuela de Periodismo de la Iglesia que refunda Ángel Herrera Oria y que años después se extiende a Barcelona y Valencia; en 1964 la sección de Periodismo que abre la Universidad de La Laguna en Canarias; e igualmente en Barcelona existió, entre 1952 y 1963, una sección de la Escuela Oficial de Periodismo.

Pasado este primer período académico en su fase embrionaria de institutos y escuelas, en 1970 la Ley General de Educación, aprobada por unanimidad, abre la puerta universitaria a los estudios de Periodismo. En 1971 el Ministerio de Educación y Ciencia aprueba los Estudios de Periodismo y demás medios de Comunicación Social en la Universidad ${ }^{1}$ una regulación que supone el origen de las nuevas Facultades de Periodismo en España y que se traduce en la creación de las pioneras: la Facultad de Ciencias de la Información de la Universidad Complutense de Madrid y la Facultad de Ciencias de la Comunicación de la Universidad Autónoma de Barcelona, al tiempo que el Instituto de Navarra se transforma en Facultad de Ciencias de la Información.

1 Boletín Oficial del Estado (B.O.E.), Nº 14 de septiembre de 1971. 
En los años 80 se crearon otros centros de enseñanza superior que experimentaron un fuerte despliegue a partir de $1991^{2}$ cuando se establece el título universitario oficial de Licenciado en Periodismo y se aprueban las directrices generales propias para organizar sus planes de estudios. Desde entonces el número de centros universitarios públicos y privados ha crecido de forma exponencial en todo el territorio español hasta sumar una oferta actual de 35 Facultades que ofrecen la titulación específica de Periodismo en el curso 2013-2014.

Tras más de 40 años de esta titulación universitaria, el mapa español cuenta en todos sus puntos cardinales con centros que ofrecen la titulación de Periodismo. Unas Facultades nacieron como Ciencias de la Información y otras como Ciencias de la Comunicación, unidas o separadas de las titulaciones hermanas de Comunicación Audiovisual, Publicidad y Relaciones Públicas, con una variación de entre 4 y 5 años de carrera.

La oferta actual de la enseñanza de Periodismo en las Universidades españolas se presenta a continuación (Tabla 1) ordenada según las fechas en las que incorporan los estudios de Periodismo; se señala pues su origen, ubicación geográfica y su titularidad pública, dependiente del Gobierno, o privada, incluyendo, si lo tiene, su carácter religioso:

TABLA 1

Universidades españolas que ofrecen la titulación de Periodismo (1971-2014) ${ }^{3}$

\begin{tabular}{|l|l|l|l|}
\hline ORIGEN & UNIVERSIDAD & PROVINCIA & TIPO \\
\hline 1971 & COMPLUTENSE & MADRID & PÚBLICA \\
\hline 1971 & NAVARRA & PAMPLONA & PRIVADA-RELIGIOSA \\
\hline 1972 & AUTÓNOMA & BARCELONA & PÚBLICA \\
\hline 1981 & PAÍS VASCO & BILBAO & PÚBLICA \\
\hline
\end{tabular}

2 Real Decreto 1428/1991 de 30 de agosto (publicado en el B.O.E. $N^{\circ} 243$, de 10 de octubre de 1991).

3 El listado, de elaboración propia, se ha realizado a partir de la información contrastada por mail y teléfono con algunas Facultades; de la consulta de la Agencia Nacional de Evaluación de la Calidad y Acreditación, ANECA (http://srv.aneca.es/ListadoTitulos/busqueda-titulaciones); del Libro Blanco Títulos de Grado en Comunicación de ANECA) y de la web del Ministerio de Educación (www. educación.es) y el Registro de Universidades (https://www.educacion.gob.es/ruct/consultaestudios. action?actual $=$ estudios)

Del Listado de la ANECA se excluyen de este análisis la Universidad de Lleida porque ofrece 'Grado en Comunicación y Periodismo Audiovisual' y la Universidad Tecnología y Empresa de Madrid cuya información oficial es inexistente. La ANECA no incluye los Grados de Periodismo del Centro Universitario Villanueva (adscrito a la Universidad Complutense), ni el Centro Eusa (adscrito a la Universidad de Sevilla) que tampoco se recogen en este análisis porque ofrecen los mismos planes de estudios que las Universidades a las que están adscritos. 


\begin{tabular}{|c|c|c|c|}
\hline 1988 & PONTIFICIA & SALAMANCA & PRIVADA-RELIGIOSA \\
\hline 1988 & LAGUNA & TENERIFE & PÚBLICA \\
\hline 1989 & SEVILLA & SEVILLA & PÚBLICA \\
\hline 1991 & $\begin{array}{l}\text { SANTIAGO DE } \\
\text { COMPOSTELA }\end{array}$ & SANTIAGO & PÚBLICA \\
\hline 1992 & MALAGA & MALAGA & PÚBLICA \\
\hline 1992 & POMPEU FABRA & BARCELONA & PÚBLICA \\
\hline 1993 & SAN PABLO CEU & MADRID & PRIVADA-RELIGIOSA \\
\hline 1994 & RAMÓN LLULL & BARCELONA & PRIVADA \\
\hline 1995 & ANTONIO DE NEBRIJA & MADRID & PRIVADA \\
\hline 1995 & EUROPEA DE MADRID & MADRID & PRIVADA \\
\hline 1996 & CARLOS III & MADRID & PÚBLICA \\
\hline 1997 & $\begin{array}{l}\text { INTERNACIONAL } \\
\text { CATALUNA }\end{array}$ & BARCELONA & PRIVADA-RELIGIOSA \\
\hline 1997 & CATÓLICA SAN ANTONIO & MURCIA & PRIVADA-RELIGIOSA \\
\hline 2000 & REY JUAN CARLOS & MADRID & PÚBLICA \\
\hline 2000 & CAMILO JOSÉ CELA & MADRID & PRIVADA \\
\hline 2000 & UNIVERSIDAD DE VALENCIA & VALENCIA & PÚBLICA \\
\hline 2000 & CEU CARDENAL HERRERA & VALENCIA & PRIVADA-RELIGIOSA \\
\hline 2002 & FRANCISCO DE VITORIA & MADRID & PRIVADA-RELIGIOSA \\
\hline 2002 & MIGUEL DE CERVANTES & VALLADOLID & PRIVADA \\
\hline 2002 & UNIVERSIDAD DE VIC & BARCELONA & PRIVADA \\
\hline 2002 & MURCIA & MURCIA & PÚBLICA \\
\hline 2003 & VALLADOLID & VALLADOLID & PÚBLICA \\
\hline 2003 & ABAT OLIBA CEU & BARCELONA & PRIVADA-RELIGIOSA \\
\hline 2004 & ROVIRA I VIRGILI & TARRAGONA & PÚBLICA \\
\hline 2005 & SANJORGE & ZARAGOZA & PRIVADA-RELIGIOSA \\
\hline 2005 & MIGUEL HERNÁNDEZ & ELCHE-ALICANTE & PÚBLICA \\
\hline 2005 & ISLAS BALEARES (CESAG) & BALEARES & $\begin{array}{l}\text { PÚBLICA-PRIV.- } \\
\text { RELIG. }\end{array}$ \\
\hline 2008 & ZARAGOZA & ZARAGOZA & PÚBLICA \\
\hline
\end{tabular}




\begin{tabular}{|l|l|l|l|}
\hline 2009 & $\begin{array}{l}\text { UIVERSIDAD A DISTANCIA } \\
\text { (UDIMA })\end{array}$ & MADRID & PRIVADA \\
\hline 2009 & JAIME I DE CASTELLÓN & CASTELLÓN & PÚBLICA \\
\hline 2010 & CASTILLA LA MANCHA & CUENCA & PÚBLICA \\
\hline
\end{tabular}

Fuente: elaboración propia

\section{La oferta y la demanda en las aulas}

Una vez analizada en cifras la oferta educativa de Periodismo se constata, pues, cómo la formación periodística ha adquirido en cuatro décadas una presencia abrumadora en el ámbito universitario y se encuentra presente en casi todas las Comunidades Autónomas. La titulación universitaria específica de Periodismo se ofrece, en la actualidad, en 35 centros repartidos en toda España. Tras el arranque inicial de los años 70 y 80, la oferta creció exponencialmente en la década de los 90 con 10 nuevas Facultades de las que, la mayoría -6 de ellas- son de carácter privado. En la primera década del 2000, lejos de detenerse, la inauguración de Facultades de Periodismo registró su mayor apogeo con 17 nuevas ofertas. Del total, 19 son públicas (54\%) y 16 privadas $(46 \%)$.

El interés y evidente incremento tanto de la oferta como de la demanda de estos estudios ha permitido recuperar "el tiempo perdido" en la formación universitaria de periodistas españoles en cuanto a disponibilidad geográfica de Facultades. En la actualidad, de las 17 Comunidades Autónomas, únicamente quedan fuera cuatro de la oferta específica de Periodismo: Asturias, Cantabria, Extremadura y La Rioja. El resto, 13 Comunidades, incluyen en sus programas Periodismo con la titulación de Licenciatura o de Grado en el presente curso. La mayor concentración de oferta se da en Madrid y en Cataluña con 10 y 7 facultades respectivamente. Hay provincias de tamaño medio donde conviven dos universidades, pública y privada, como es el caso de Valladolid, Zaragoza o Murcia. En algunas Universidades se ofrecen dobles titulaciones en las que se combinan las diferentes ofertas en Comunicación con otras como Historia, Ciencias Políticas, Derecho, Economía.

Y en consonancia con esta realidad, la demanda de alumnos también sigue en aumento. Por las 35 Facultades de Periodismo españolas han pasado ya más de 74.900 licenciados y ahora también egresados lo que supone una media de casi 1.700 periodistas que cada año salen al mercado laboral. Este alto nivel de demanda universitaria contrasta con los datos que refleja la crisis actual que sufre el sector en España desde que comenzara la caída económica en 2008. El paro registrado del colectivo de periodistas asciende a casi 27.500 personas (APM, 2012), una cifra que se ha triplicado en apenas un año y sobre la que se profundiza más adelante. 
La profesión periodística aún padece las consecuencias del intrusismo puesto que a pesar de la expansión de los centros universitarios y la importante demanda de estudiantes, para ejercer la profesión no se exige ningún título específico ni colegiación, lo que favorece el acceso libre de no titulados. Pero ante la usencia de un estatuto regulador que genera discrepancias entre los profesionales, la Universidad va ganando su batalla y en España, más del $80 \%$ de los periodistas ya cuenta con una titulación universitaria (APM, 2012). Una realidad que, poco a poco va imponiéndose en las redacciones.

\section{ADAPTACIÓN AL ESPACIO EUROPEO DE EDUCACIÓN SUPERIOR (EEES)}

La implantación del nuevo plan de convergencia educativa europea -conocido como "Plan Bolonia" y oficialmente, Espacio Europeo de Educación Superior (EEES) - en el curso 2010-2011, supone un desafío en la formación universitaria española y de los 47 países miembros implicados en esta reforma que trata de homogeneizar títulos y sistemas de evaluación. España acaba de pasar por el proceso de implantación y aún no existe suficiente perspectiva para analizar los resultados pedagógicos y, mucho menos, de adaptación al mercado laboral. El cambio académico supone el paso de la Licenciatura (que desaparece de forma progresiva en 2015) al nuevo título de Grado que implica una modificación en todos los planes de estudio incluidos, por supuesto, los de Periodismo.

La nueva titulación se fraguó hace varias décadas en Europa y de esta han quedado ya unas fechas claves, como la de 1988 con la firma de la Carta Magna de las Universidades que suponía el compromiso inicial al cambio de los rectores de universidades europeas. Le siguió la Declaración de la Sorbona (25 de mayo de 1998) en una reunión que contó con la intervención de ministros de Educación de cuatro países europeos: Alemania, Italia, Francia y Reino Unido (que pertenecen al G7, el grupo que conforman las naciones más ricas del mundo). En 1999 se firmó la Declaración de Bolonia, en la ciudad italiana que da nombre al Plan, donde 29 ministros de Educación europeos rubricaron la citada Declaración de Bolonia que ha dado lugar a la creación del Espacio Europeo de Educación Superior (EEES).

Como se ha indicado anteriormente, se trata de la convergencia de los sistemas universitarios de 47 países europeos, con el fin de homogeneizar la enseñanza universitaria y sus titulaciones mediante la adaptación conjunta de los planes de estudio de Grado. Una unificación educativa, al estilo de la moneda única del euro, que se sostiene en tres pilares: una mayor movilidad de estudiantes que pueden cursar su carrera en distintos países y la libre circulación de los profesionales de Europa con sus títulos homologados; todo ello a través del tercer pilar de la reforma, la aplicación de un nuevo método docente en el que las Universidades tendrán casi 
total libertad para elaborar su oferta educativa. "La independencia y autonomía de las Universidades asegura que los sistemas de educación superior e investigación se adapten continuamente a las necesidades cambiantes, las demandas de la sociedad y los avances en el conocimiento científico” (Ministerio de Educación, 1999).

El nuevo sistema incluye, además, novedades tanto en la metodología docente como en los criterios de evaluación y la programación delas asignaturas. El plan antiguo de Licenciatura basaba sus objetivos principales en la adquisición de conocimientos con la clase magistral del profesor como elemento básico de la docencia. El nuevo Grado aporta dos conceptos, además de conocimientos: habilidades y competencias. En líneas generales, los nuevos Grados se encaminan a ofrecer mayor especialización y preparación de técnicos y profesionales cualificados en tres niveles: primer ciclo de Grado, segundo ciclo de Máster y Doctorado.

El proceso de adaptación a este nuevo modelo educativo y los efectos en la formación de los periodistas aún no se han estudiado de forma profunda. Sin embargo, como una primera aproximación se puede apuntar una investigación realizada en la Universidad de Valladolid por quien suscribe este artículo, con un análisis comparativo entre los planes de estudio de la Licenciatura y del Grado en Periodismo para comprobar las variaciones en la enseñanza periodística encaminada a la convergencia europea. Los primeros resultados evidencian que se acentúa el carácter práctico de sus materias con el refuerzo de asignaturas encaminadas al adiestramiento técnico de la profesión, al tiempo que aumentan las asignaturas más especializadas en contenidos estrechamente relacionados con la titulación en detrimento de las materias de conocimientos generales y humanísticos (Sánchez, 2010). Los datos indican, entonces, que se ha adaptado la titulación, al menos en esta Universidad, al carácter más práctico y técnico que establecen las directrices del nuevo Espacio Europeo de Educación Superior (EEES), implantado obligatoriamente en 2010. Unas conclusiones iniciales que permiten aventurar resultados similares en el resto de las Facultades españolas que ofrecen la titulación de Periodismo ${ }^{4}$, puesto que todas han tenido como guía las directrices marcadas desde la Agencia Nacional de Evaluación de la Calidad y Acreditación (ANECA), el organismo español que verifica la adaptación de cada titulación universitaria al programa europeo.

4 Este análisis forma parte de la tesis doctoral que ultima la firmante de este artículo con un comparativo de los planes de estudio de Licenciatura y Grado en las 35 Facultades con titulación de Periodismo en España, al objeto de comprobar el cambio de contenidos de los estudios al aplicarse los criterios europeos del Plan Bolonia. 


\section{LA DOBLE COYONTURA ACTUAL: CRISIS DEL SECTOR Y NUEVOS MODELOS DE NEGOCIO CON CAMBIO DE PERFILES PERIODÍSTICOS}

Expuesta, hasta aquí, una aproximación al ámbito académico del Periodismo en España, se añaden ahora los principales rasgos que definen el ámbito laboral y profesional actual de los periodistas, al objeto de poder concretar más eficazmente los restos formativos actuales.

La primera década del siglo XXI se caracteriza por un cambio vertiginoso en el sector de los medios de comunicación en el que confluye una doble coyuntura: la crisis económica que sitúa el periodismo como la segunda profesión con más parados en España y la revolución tecnológica que mantiene las rutinas productivas y los modelos de negocio en un momento de indefinición, a la vez que representan nuevas oportunidades laborales por la ampliación de los perfiles periodísticos en la era digital. Un cambio estructural del sector en todas sus facetas: las rutinas productivas, la situación laboral, los ingresos por publicidad, los soportes, la relación con las fuentes y los receptores, las nuevas formas de pago, etc. El tradicional modelo informativo y de negocio se ve obligado a asumir una transformación repentina e inmediata en la que ahora está inmerso el periodismo, en particular, y la comunicación en su conjunto. Todo ello agudizado por la crisis económica, que alberga un futuro aún por definir y que quizá carezca de suficiente perspectiva aún para predecir la siguiente fase del devenir más inmediato.

En primer lugar, la crisis económica actual tiene dos claras consecuencias en los medios de comunicación españoles: el cierre de empresas y los miles de despidos de trabajadores a causa de la caída drástica de la publicidad y los cambios de usos de las audiencias con las nuevas tecnologías. Según la Federación de Asociaciones de Periodistas de España, la repercusión económica y laboral resulta tan fuerte que en apenas cinco años en España se contabilizan 10.410 despidos de trabajadores de medios de comunicación y el cierre de 86 empresas (desde noviembre de 2008 a noviembre de 2013). Se trata de la segunda profesión más afectada en el conjunto nacional, después de la construcción, sobre la que se ha apoyado durante décadas la economía española generando una bonanza ficticia o temporal que ha desembocado en la explosión de la llamada "burbuja inmobiliaria". Los medios de comunicación, beneficiados de esta etapa de grandes ingresos económicos, sufren ahora la caída de todos los sectores en un "efecto dominó" acusado principalmente en la merma de los ingresos por publicidad, tanto pública como privada, de la que el modelo periodístico tradicional ha sido excesivamente dependiente. Entre 2007 y 2011, los ingresos por publicidad comercial cayeron un $30 \%$, con especial incidencia en los medios impresos: en los diarios, el descenso fue del 47,8\%, y del 46,3\% en la revistas. La televisión perdió menos, con un descenso del 34\% (Díaz, 2011, p. 28). De igual forma, la bajada de inversión publicitaria de los organismos públicos es muy relevante, con el recorte de 
la Administración central en medios del 70,2\%, entre 2007 y 2010 (Ministerio de Presidencia, 2011).

Estos fuertes recortes, que han seguido en caída libre, resultan más problemáticos al comprobar que el traslado de la publicidad de los medios a través de Internet, no genera los beneficios esperados y mucho menos los de época de bonanza. De hecho, en España se ha tardado en notar este trasvase de anunciantes hasta el año 2012, año en que, por primera vez desde la aparición de Internet, la inversión publicitaria en medios digitales (18,3\%) supera a la inversión en medios impresos (15,6\%). Situándose en segunda posición sólo por detrás de la inversión en televisión (43\%) (Web IAB, 2013), al menos hasta la actualidad.

La crisis económica igualmente ha afectado a las audiencias, el segundo pilar en el que se ha sustentado hasta ahora el tradicional modelo de negocio de los medios de comunicación. Las nuevas tecnologías están propiciando cambios de usos de las audiencias, de manera que al desplome de lectores de papel, se unen las nuevas formas de consumo de los productos audiovisuales tradicionales. El consumidor de los medios, considerado como un antiguo receptor pasivo, ha adquirido el estatus de emisor y productor activo. Biondi, Miró y Zapata (2010) van más allá al asegurar que incluso al ciudadano ya no se le puede considerar simplemente como un consumidor, sino que se convierte en un "prosumidor", es decir, "asume los roles, de forma simultánea, de consumidor y productor de información” (2010, p. 106). Se trata de una audiencia más independiente, selectiva y dispersa a la hora de informarse.

De esta forma, frente a la caída de ingresos por las dos vías principales de la publicidad y la audiencia, los medios de comunicación ven superadas sus estructuras tradicionales y avanzan hacia nuevos modelos de negocio en lo que ha pasado a denominarse Ciberperiodismo, un término que Montiel y Villalobos (2005) definen como un "intercambio automatizado de información entre miles de personas de todo el planeta" (2005, p. 399). En este contexto se produce una fusión de los medios tradicionales en un producto interactivo y multimedia que incorpora video, audio, textos, sistemas de búsquedas, actualizaciones inmediatas, participación del usuario, etc. Un periodismo multisoporte y multilenguaje.

En esta realidad profesional de extrema turbulencia (Salaverría, 2012) las estructuras empresariales obligadas a reorganizarse renuevan una oferta multimedia para una audiencia informativamente hiperconectada a través de las nuevas tecnologías. Los cambios más relevantes de la convergencia digital abarcan desde la multiplicación de redes informativas, canales y pantallas de acceso a la información hasta "la capacidad de uso social de la misma por parte de los ciudadanos" (Campos, 2011 , p. 3). Y todo ello afecta al ámbito tecnológico, empresarial, profesional y editorial de los medios de comunicación, con la integración de nuevas herramientas, mercados y lenguajes.

En esta nueva Sociedad de la Información, el cambio de modelo de negocio 
mencionado trastoca múltiples perspectivas de gestión del sistema industrial, "desde los principios de la propiedad (protección de la obra y la producción intelectual) al propio marco del contrato social (flexibilidad, freelance, blogger, emprendedores individuales, geeks, cloud creative, open access journals, etc.)” (Campos, 2011, p. 3). Parecen no existir dudas sobre la necesidad de este cambio de negocio, pero aún los medios se encuentran en una etapa de ensayo y error porque, a pesar de saber que la transformación es irreversible e irremediable, no se sabe realmente la forma más adecuada de afrontarla. Prueba de ello es que cada medio ha empezado por analizar su realidad y adoptar aquella que mejor comulgue con su cultura organizacional (Biondi, Miró y Zapata, 2010, p. 114).

Por ahora, se puede decir que en España, estos nuevos modelos de negocio resultan incipientes. Y en las nuevas formas de gestionar las empresas periodísticas se siguen los pasos pioneros de EEUU donde sirve de referencia, por poner un ejemplo, el cambio que introduce en 2011 el diario The New York Times con un novedoso sistema de pago por consumo denominado metered paywall, con una oferta limitada de contenidos gratuitos (20 artículos al mes) y una suscripción de pago para los usuarios más intensivos y que cosecha en sus inicios 324.000 suscriptores digitales, una cifra que cumplía las mejores expectativas (Salaverría, 2012, p. 163). Un modelo que, con algunas diferencias, acaba de incorporar el segundo periódico en España, El Mundo, a través de su plataforma digital Orbyt que ofrece un sistema de pago similar basado en una determinada cantidad de contenido a consumir, con distintas franjas de pago, desde octubre de 2013 e incluso con una nueva edición vespertina en su web informativa www.elmundo.es.

La mencionada doble coyuntura de crisis económica y cambios de modelo de negocio auspiciados por las nuevas tecnologías conlleva, igualmente, variaciones muy relevantes en las rutinas productivas de los periodistas con una mayor variedad de tareas y de perfiles laborales.

No hay que confundirse, "el trabajo de siempre continúa siendo en esencia el mismo” (Díaz, 2002, p. 178), es decir que las funciones del periodista de informar, analizar, interpretar y difundir la información no varían en su esencia. Y, en especial, debe seguir inalterable su función de servicio público, en el sentido de hacer efectivo el Derecho a la Información de los ciudadanos (artículo 20 de la Constitución Española, 1978) que contribuye al desarrollo democrático y al progreso social que sustenta la relevancia de esta profesión.

Sentadas estas bases, lo que sí ha cambiado en el trabajo del informador profesional son sus rutinas diarias con multitud de nuevas tareas. El periodista que trabaja con los medios digitales, el ciberperiodista o periodista digital es un profesional que requiere habilidades y competencias particulares que no han de limitarse al uso de las herramientas de software, sino que "ha de ser capaz de dominar una serie de tareas que engloban: el acceso, el manejo, la integración, la creación y la evaluación de los 
mensajes informativos on-line" (Tejedor, 2007,p.407). Ya no sólo se dedica a producir contenidos, sino que se potencia su faceta de "gestor de información" ante la nueva sobreabundancia de fuentes, datos y posibles noticias, lo que requiere un perfil más complejo y formativamente exigente. El periodista actual debe contar con destrezas suficientes para trabajar en diferentes soportes (multimedia), manejando los lenguajes escrito y audiovisual (multilenguaje) en una realidad periodística que ha dejado de ser lineal, tanto en la información que ofrece como en el lenguaje que utiliza. En palabras de Masip \& Micó (2009, p. 93), el periodista multimedia es sinónimo de informador polivalente y versátil desde diferentes perspectivas: polivalencia mediática, en el sentido de que debe producir contenidos para distintos soportes; polivalencia tecnológica porque se hace cargo de todo el proceso productivo; y polivalencia temática porque debe ahora tratar noticias de distintos ámbitos o secciones.

En definitiva, en España, como en el resto de países, los medios de comunicación están inmersos en el reto de superar su crisis publicitaria, encontrar nuevos modelos de negocio que atraigan a las audiencias cambiantes y asumir e integrar los nuevos perfiles del periodista multimedia. Una labor, de la que la Universidad no puede sentirse ajena, como se expone a continuación en el relato de los retos formativos que debe asumir.

\section{PRÓXIMOS DESAFÍOS FORMATIVOS: LA INCORPORACIÓN DE LAS TECNOLOGÍAS Y EL RECICLAJE PERMANENTE}

Las necesidades formativas de un periodista se encuentran estrechamente ligadas a la propia realidad cambiante del trabajo en las redacciones, gabinetes, instituciones o empresas y por la evolución de la sociedad en la que ejerce la profesión. La revolución de las nuevas tecnologías representa también un gran desafío dentro de las aulas entendiendo que las herramientas digitales representan nuevas oportunidades laborales para los periodistas que requieren una mayor preparación y una adaptación formativa. La Universidad debe involucrarse en este reto.

La formación del periodista multimedia se convirtió en una preocupación académica en España a finales del siglo XX cuando profesores como Salaverría, Díaz Noci, Meso o Nosty, entre otros, comenzaron a criticar la lentitud de las facultades en la incorporación de las nuevas tecnologías y el consiguiente desfase en los planes de estudio con la realidad de la información digital (Meso, 2003).

La mayoría de las facultades españolas con titulación de periodismo han ido incorporando en sus planes de estudio la formación tecnológica y las enseñanzas del llamado ciberperiodismo, periodismo electrónico o multimedia. Desde que en 1994 se diera el primer paso en este sentido con la fundación del Laboratorio de Comunicación Multimedia en Navarra y con la primera asignatura sobre periodismo 
e Internet, muchas otras universidades han ido incorporando a sus licenciaturas y postgrados estudios sobre periodismo digital. Paralelamente, se han publicado las primeras monografías y tesis doctorales sobre distintos aspectos de esta nueva realidad (Salaverría, 2000, p. 2). Y, más recientemente, se puede decir que todas las facultades españolas han aprovechado la adaptación de sus programas al modelo de convergencia europeo, explicado anteriormente, para incluir estas nuevas disciplinas propias del periodismo digital y multimedia en la titulación de grado.

Pero este cambio no implica únicamente incorporar herramientas técnicas, sino responder al desafío de propiciar una formación integral que permita a los futuros periodistas desenvolverse en un nuevo mercado polivalente, cambiante y multimedia.

El reto actual se encuentra, por tanto, en dirimir un nuevo debate en la enseñanza periodística con dos modelos diferenciados: eldelos periodistasformados para medios digitales, a los que se refuerza la capacitación instrumental; y el de los periodistas que reciben una formación para la era digital, a quienes, sin desdeñar la parte técnica, se les prepara para convertirse en profesionales versátiles y con criterio; es decir, con la formación teórica suficiente y amplia como para responder informativamente en cualquier soporte ante una realidad en constante cambio que requiere mayores dosis de interpretación (Salaverría, 2000). La enseñanza simplemente técnica del periodismo se limita a "entrenar al profesional pero no formar al hombre" y la enseñanza actual debe pasar por el refuerzo del criterio del periodista que selecciona, analiza y transmite la información (Aguirre, 1988, p. 204). Debe ser profunda y con la prioridad del criterio periodístico, independientemente del soporte.

En definitiva, la universidad española debe responder al reto de formar periodistas más allá de la enseñanza instrumental del manejo de un navegador, diferentes bases de datos o webs y blogs. La instrucción técnica es una herramienta que facilita las repuestas en un proceso informativo nuevo, en el que se deben tener en cuenta los nuevos perfiles multimedia del periodista que debe ser capaz, como ya se ha dicho, de gestionar una mayor cantidad de información, elaborarla para cualquier tipo de soporte y asumir nuevas formas de multilenguaje asumida por el hipertexto.

Así, entre los múltiples aspectos que debe asimilar la formación periodística en la era digital se encuentran las nuevas teorías sobre formas narrativas digitales, entendidas como narraciones que aprovechan las posibilidades de todos los formatos y que ofrecen una exposición multimedia para el ciberlector que ya no busca una información lineal.

Los periodistas del futuro, los que ahora se están formando en las aulas universitarias y quienes lo harán en los próximos años, no sólo deberán conocer las implicaciones de hipertextualidad, multimedialidad e interactividad que caracterizan a los medios digitales, sino que deberán ser capaces de dar un salto más allá y convertirlas en una estrategia creativa en aquellas producciones que así lo requieran, como es el caso de los reportajes multimedia (Palau, 2010, p. 142). 
Este reto obliga a apostar por una formación académica en la que se combinen las habilidades técnicas de los distintos formatos con los procesos narrativos que demanda ya un lector digital que, más allá de la búsqueda de datos ordenados y desligados, valore la calidad expositiva de los mismos. Un ciberlector en busca de la comprensión narrativa que le permite acceder a fenómenos complejos, como los que tradicionalmente suele abordar el reportaje, un género periodístico aún por explorar en el entorno digital. Y el reto de adaptación formativa a las nuevas tecnologías pasa igualmente por considerar a los periodistas multimedia, no sólo como creadores de contenidos, sino también como gestores de información.

Esta adaptación a nuevos soportes, herramientas y creación de nuevas narrativas va ligada directamente a un segundo reto o desafío que se debe afrontar en la enseñanza periodística, como es el de responder a la necesidad de una formación más exigente, en la que se dé un equilibrio entre aspectos teóricos y prácticos. Una combinación que se une al ineludible objetivo de propiciar en los alumnos el espíritu de formación individual y permanente que incluye el necesario reciclaje de los periodistas en activo y la actualización constante de los recién titulados porque las rutinas profesionales y los hábitos de los receptores siguen su proceso cambiante.

Nunca antes se le había exigido tanto a los profesionales de la Comunicación. Cada vez se perfila con más fuerza una característica de la nueva era de la información: la necesidad de la formación continua. Desde ahora todos somos aprendices. Un informe del Grupo de Expertos sobre Aspectos Sociales y Societarios de la Sociedad de la Información (HLG) para la Unión Europea contempla, entre sus conclusiones, la nueva sociedad como una sociedad del aprendizaje, basada en el saber-hacer y en la sabiduría de las personas, no en la información en las máquinas (Meso, 2003, p. 8).

Precisamente este desafío se contempla ya en los nuevos planes de estudio europeos que recogen como objetivo fundamental la formación constante de los universitarios con el denominado Marco Europeo de Cualificaciones para el aprendizaje permanente (EQF-MEC). Un instrumento que permite acreditar el proceso formativo del alumno antes y después de la universidad y que sirve como carta de presentación laboral en los diferentes países europeos.

En definitiva, la llamada Sociedad del Conocimiento y de la Información, la defensa del "conocimiento abierto" y del Open Data facilitan el trabajo de los periodistas, al tiempo que les exige una constante revisión y actualización de sus técnicas de trabajo y de sus conocimientos.

\section{DISCUSIÓN Y CONCLUSIONES}

La revisión de la enseñanza periodística en España y de la realidad profesional actual permite extraer varias conclusiones en torno a los actuales desafíos formativos. 
En primer lugar, la formación de los periodistas españoles ha estado influida por el control de la dictadura Franquista y de la visión del periodismo como oficio en determinados sectores. Ambos condicionantes han retrasado la incorporación de los estudios de periodismo a la universidad y su correspondiente desarrollo científico.

En segundo lugar, sigue abierto hoy el viejo debate entre los defensores y detractores de la formación periodística en la Universidad pero la realidad se ha impuesto y, a pesar de la falta de regulación y la no exigencia de un título para ejercer la profesión, la demanda y la oferta de los estudios universitarios de periodismo se ha multiplicado exponencialmente en sus cuatro décadas de enseñanza superior con 35 facultades que imparten la titulación (1971-2013) y con más de 70.000 licenciados.

En tercer término, la realidad indica que la universidad debe mostrarse atenta a los resultados de los primeros análisis sobre los efectos programáticos y pedagógicos de los nuevos planes de estudio adaptados al Espacio Europeo de Educación Superior desde el curso 2010-2011. Las investigaciones aún escasas evidencian que algunos programas de enseñanza periodística, como muestra el estudio clínico de la Universidad de Valladolid, han incrementado su carácter técnico-práctico y el contenido de materias especializadas en la profesión, en detrimento de la formación humanística y teórica. Un cambio acorde con el planteamiento más práctico y especializado que propugna el nuevo modelo educativo europeo cuyos resultados pedagógicos y laborales requieren mayor perspectiva temporal.

En quinto lugar, el ámbito profesional del periodismo vive en la actualidad una doble coyuntura de crisis económica y revolución tecnológica con unas consecuencias muy concretas interrelacionadas entre sí: bajada de los ingresos por publicidad, cambio de usos de las audiencias, la necesidad de nuevos modelos de negocio y el cambio de rutinas periodísticas con el aumento de perfiles profesionales.

En sexto término, la universidad española afronta el reto de adaptarse a estos cambios tecnológicos del sector con la implantación de materias relacionadas con las nuevas herramientas del Ciberperiodismo desde un punto de vista transversal y multidisciplinar. En este proceso surgen dos modelos formativos, el que prima la enseñanza técnica más instrumental o el de la formación integral del periodista que conoce las herramientas que le permitirán afrontar con el mayor rigor posible el nuevo mercado laboral polivalente, cambiante y multimedia. Los expertos alertan de la necesidad de implantar y extender este segundo modelo que permita al futuro periodista adquirir conocimientos suficientes y potenciar el criterio periodístico necesario para interpretar correctamente la realidad actual más compleja y ofrecer un mejor periodismo interpretativo con el objetivo de cumplir con su invariable función social de garantizar el Derecho a la Información de los ciudadanos.

Y, en séptimo y último lugar, tanto los centros universitarios como los propios profesionales de la información deben fomentar la formación permanente $y$ autodidacta en un contexto tecnológico cambiante y en una realidad informativa compleja en constante evolución. 


\section{Referencias bibliográficas}

Aguinaga, E. (1980). Periodismo profesión. Madrid: Fragua.

Aguinaga, E. (1984). Epistemología del ejercicio periodístico. Los estudios de periodismo y su proyección profesional. Madrid: Universidad Complutense.

Aguirre, M. (1988). El deber de formación en el informador. Pamplona: Eunsa.

Agencia Nacional de Evaluación de la Calidad y Acreditación, ANECA. (s.f.). Titulaciones de Grado en Periodismo. Extraído el 10 de enero de 2012, desde http://srv.aneca.es/ListadoTitulos/busqueda-titulaciones

Agencia Nacional de Evaluación de la Calidad y Acreditación. (2005). Libro Blanco. Título de Grado en Comunicación. Extraído el 3 de febrero de 2010, desde http://www.aneca.es/media/150336/libroblancocomunicaciondef.pdf

Asociación de la Prensa de Madrid.(2012). Informe Anual dela profesión periodística. Madrid: Asociación de la Prensa de Madrid.

Beneyto, J. (1958). España. En UNESCO, La formación de periodistas. Estudio mundial sobre la preparación del personal de la información (pp. 199-202). París: UNESCO.

Benito, A. (1967). Evolución de los estudios de Periodismo en el mundo. Ciencia y enseñanza del Periodismo. Cuadernos de Trabajo, 11, 13-36.

Benito, A. (1982). Fundamentos de Teoría General de la Información. Madrid: Pirámide.

Biondi, S., Miró, S. y Zapata, E. (2010). Derribando muros. Periodismo 3.0: oferta y demanda de comunicación en el Perú de hoy. Lima: El Comercio.

Campos, F. (2011). Las empresas de medios de comunicación revisan y amplían sus modelos de negocio. Razón y palabra, 74.

Díaz, B. (2011). Libro negro del periodismo en España. Madrid: Asociación de la Prensa de Madrid.

Díaz, J. (2002). La Escritura Digital: Hipertexto y Construcción Del Discurso Informativo en el Periodismo Electrónico. Gipuzkoa: Universidad del País Vasco.

Federación de Asociaciones de Periodistas de España, FAPE. (2010). Observatorio de la crisis. Extraído el 14 de noviembre de 2013, desde http:// www.fape.es/

Gordón, M. (1991). La enseñanza del Periodismo en el mundo occidental. Estudio Comparado de tres escuelas. Tesis para optar al grado de Doctor, Universidad Complutense de Madrid, Madrid.

Graña, M. (1927). Escuelas de Periodismo. Conferencia pronunciada en la Sociedad de Estudios Vasca.

Humanes, M. L. (1998). La profesión periodística en España. Zer. Revista de estudios de comunicación, 4. 
IAB (Interactive Advertising Bureau). (2012). Estudio de inversión publicitaria del primer semestre de 2012. Extraído el 9 de septiembre de 2013, desde http:// www.iabspain.net/noticias/por-primera-vez-la-inversion-publicitaria-enmedios-digitales-183-supera-a-la-de-medios-impresos-156/

Iglesias, F. (1984). Guía de los Estudios Universitarios. Pamplona: Eunsa.

Masip, P. y Micó, J.L. (2009). El periodista polivalente en el marco de la convergencia empresarial. Quaderns del CAC, 31-32, 91-99.

Meso, K. (2003). La formación del periodista digital. Chasqui, Revista Latinoamericana de Comunicación, 84, 4-11.

Ministerio de Educación. (2003). La integración del sistema universitario español en el Espacio Europeo de Enseñanza Superior. Documento marco. Extraído el 16 de junio de 2010, desde http://www.eees.es/es/documentacion

Ministerio de Educación. (1999). Declaración de Bolonia. Extraído el 13 de junio de 2010, desde http://www.eees.es/es/documentacion

Ministerio de la Presidencia. (2011). Informe de Publicidad y Comunicación Institucional 2010. Extraído el 4 de septiembre de 2013, desde http:// www.lamoncloa.gob.es/NR/rdonlyres/6F9F7C99-80EE-40C9-862B9534075E3128/160589/Informe2010.pdf

Montiel, M. y Villalobos, F. (2005). La enseñanza del periodismo en el siglo XXI: un desafío entre lo impreso y lo digital. Telos, 7(3), 397-411.

Palau, D. (2010). El reto de formar narradores digitales. En Sierra, J. y Cabezuelo, F. (Coords.), Competencias y perfiles profesionales en los estudios de Ciencias de la Comunicación (pp. 134-143). Madrid: Fragua.

Real, E. (2004). Formación y ejercicio profesional del periodista en la España del siglo XXI dentro del marco de la Unión Europea. Tesis para optar al grado de Doctor, Universidad Complutense de Madrid, Madrid.

Sáiz, M. D. y Seoane, M.C. (1996). Historia del Periodismo. Madrid: Alianza Editorial.

Salaverría, R. (2000). Criterios para la formación de periodistas en la era digital. Trabajo presentado en el I Congreso Nacional de Periodismo Digital, Enero, Huesca.

Salaverría, R. (2012). Extrema turbulencia en los medios en 2011. Anuario ThinkEPI, v. 6. Extraído el 30 de mayo de 2013, desde http://dspace.unav.es/dspace/ bitstream/10171/21940/1/Anuario ThinkEPI 2012 pp 161-165 MEDIOS-Salaverria.pdf

Sánchez, P. (2010). Una aproximación a la formación universitaria del periodista en España. Análisis comparativo entre la Licenciatura y el Grado de Periodismo en la Universidad de Valladolid (2010-2011). Tesina defendida en la Universidad de Valladolid (inédita).

Sierra, J. y Cabezuelo, F. (Coord.). (2010). Competencias y perfiles profesionales en los estudios de Ciencias de la Comunicación. Madrid: Fragua. 
Sierra, J. (Coord). (2010): Los estudios de Ciencias de la Comunicación en el EEES. Madrid: Fragua.

Tejedor, S. (2007). La enseñanza del ciberperiodismo: enseñary aprender periodismo on-line. En Gómez, B. y Hernández, S. (Eds.), Estudios de Periodística XIII. La Periodística como disciplina universitaria: balance y perspectivas (pp. 357363). Pamplona: Universidad de Navarra.

Videla, J.J. (2002). La formación de los periodistas en España: perspectiva histórica y propuestas de futuro. Tesis para optar al grado de Doctor, Universidad Complutense de Madrid, Madrid.

Vigil y Vázquez, M. (1987). El Periodismo Enseñado. De la Escuela de 'El Debate' a Ciencias de la Información. Barcelona: Mitre. 